Article

\title{
Extracts Obtained from Pterocarpus angolensis DC and Ziziphus mucronata Exhibit Antiplasmodial Activity and Inhibit Heat Shock Protein 70 (Hsp70) Function
}

\author{
Tawanda Zininga ${ }^{1}$, Chinedu P. Anokwuru ${ }^{2}$, Muendi T. Sigidi ${ }^{3}$, Milingoni P. Tshisikhawe ${ }^{4}$, \\ Isaiah I. D. Ramaite ${ }^{2}$, Afsatou N. Traoré ${ }^{3}$ (D), Heinrich Hoppe ${ }^{5}$, Addmore Shonhai ${ }^{1, *}$ \\ and Natasha Potgieter ${ }^{3,6}$ \\ 1 Biochemistry Department, School of Mathematical and Natural Sciences, University of Venda, \\ Private Bag X5050, 0950 Thohoyandou, South Africa; tzininga@gmail.com \\ 2 Chemistry Department, School of Mathematical and Natural Sciences, University of Venda, \\ Private Bag X5050, 0950 Thohoyandou, South Africa; anokwuruchi@gmail.com (C.P.A.); \\ isaiah.ramaite@univen.ac.za (I.I.D.R.) \\ 3 Microbiology Department, School of Mathematical and Natural Sciences, University of Venda, \\ Private Bag X5050, 0950 Thohoyandou, South Africa; muendi.sigidi@yahoo.com (M.T.S.); \\ afsatou.traore@univen.ac.za (A.N.T.); natasha.potgieter@univen.ac.za (N.P.) \\ 4 Botany Department, School of Mathematical and Natural Sciences, University of Venda, Private Bag X5050, \\ 0950 Thohoyandou, South Africa; peter.tshisikhawe@univen.ac.za \\ 5 Department of Biochemistry and Microbiology, Rhodes University, P.O. Box 94, \\ Grahamstown 6140, South Africa; h.hoppe@ru.ac.za \\ 6 School of Mathematical and Natural Sciences, University of Venda, Private Bag X5050, 0950 Thohoyandou, \\ South Africa \\ * Correspondence: addmore.shonhai@univen.ac.za; Tel.: +27-15-962-8723; Fax: +27-15-962-8002
}

Received: 19 June 2017; Accepted: 18 July 2017; Published: 28 July 2017

\begin{abstract}
Malaria parasites are increasingly becoming resistant to currently used antimalarial therapies, therefore there is an urgent need to expand the arsenal of alternative antimalarial drugs. In addition, it is also important to identify novel antimalarial drug targets. In the current study, extracts of two plants, Pterocarpus angolensis and Ziziphus mucronata were obtained and their antimalarial functions were investigated. Furthermore, we explored the capability of the extracts to inhibit Plasmodium falciparum heat shock protein 70 (Hsp70) function. Heat shock protein 70 (Hsp70) are molecular chaperones whose function is to facilitate protein folding. Plasmodium falciparum the main agent of malaria, expresses two cytosol-localized Hsp70s: PfHsp70-1 and PfHsp70-z. The PfHsp70-z has been reported to be essential for parasite survival, while inhibition of PfHsp70-1 function leads to parasite death. Hence both PfHsp70-1 and PfHsp70-z are potential antimalarial drug targets. Extracts of P. angolensis and Z. mucronata inhibited the basal ATPase and chaperone functions of the two parasite Hsp70s. Furthermore, fractions of P. angolensis and Z. mucronata inhibited P. falciparum 3D7 parasite growth in vitro. The extracts obtained in the current study exhibited antiplasmodial activity as they killed P. falciparum parasites maintained in vitro. In addition, the findings further suggest that some of the compounds in P. angolensis and Z. mucronata may target parasite Hsp70 function.
\end{abstract}

Keywords: antimalarial activity; Hsp70; molecular chaperone; Pterocarpus angolensis; Ziziphus mucronata 


\section{Introduction}

Malaria remains a major killer disease with devastating outcomes, particularly in children and pregnant mothers. Although currently malaria is a treatable disease, it is of concern that the population of parasites that are resistant to the most reliable antimalarial treatment (artemisinin-based combination therapies) is growing [1]. It is against this background that it is important to search for alternative effective antimalarial drugs. In addition, it is also important to identify novel antimalarial drug targets.

Heat shock proteins (Hsps) are molecules that facilitate protein folding (molecular chaperones) [2]. The Hsps play a central role in protein quality control and as such they have been proposed as druggable candidates in infectious diseases, among them malaria [3]. The Hsp70 members constitute one of the most ubiquitous molecular chaperone families. Structurally, Hsp70s are constituted by an N-terminal nucleotide binding (ATPase) domain and a substrate binding domain (SBD) located at the C-terminus. These two domains are connected by a linker which facilitates the allosteric signals between the two domains. The Hsp70s exhibit low basal ATPase activity [4]. In the ATP-bound state Hsp70 exhibits low affinity for substrates, leading to substrate release. On the other hand, in the ADP-bound state, Hsp70 possesses higher affinity for substrate binding [5]. The substrate dwell time is influenced by the nucleotides bound to Hsp70s. The Hsp70 functional cycle is regulated by co-chaperones amongst them, Hsp40 proteins. The Hsp40s interact with Hsp70s through their conserved J-domain and activates the Hsp70 ATPase activity [6]. As bi-functional molecules, Hsp40s are also involved in the substrate recruitment to Hsp70.

The Hsp110 proteins are distant family members of Hsp70s, which are constituted by a highly conserved ATPase domain and a less conserved SBD. The Hsp110s have an insertion on the SBD making them larger molecules of average molecular-weight size of $110 \mathrm{kDa}$, compared to canonical Hsp70s whose sizes are generally around $70 \mathrm{kDa}$. The Hsp110 proteins function as nucleotide exchange factors (NEF) for canonical Hsp70s [7].

The main agent of malaria, Plasmodium falciparum, expresses six members of the Hsp70 family of which two are cytosol-localized: PfHsp70-1 and PfHsp70-z [8]. Parasite Hsp70s have been proposed as potential antimalarial drug targets [3,9]. We previously reported that both PfHsp70-1 and PfHsp70-z exhibit independent chaperone functions [10-14]. The PfHsp70-1 is a canonical Hsp70, as it is closely related to the Escherichia coli Hsp70 homolog (DnaK). On the other hand, PfHsp70-z belongs to the Hsp110 family. The Hsp110s exhibit independent chaperone activity apart from serving as NEFs of canonical Hsp70s [7]. We previously reported that PfHsp70-1 interacts with PfHsp70-z, and for this reason we think that PfHsp70-z acts as an independent molecular chaperone and may also serve as a NEF for PfHsp70-1 [13]. The two proteins are thought to function in cooperation towards facilitating protein folding to facilitate survival of malaria parasites. Protein quality control is important for the survival of the parasite, since $24 \%$ of the $P$. falciparum proteome is composed of asparagine repeat rich proteins which tend to aggregate under stress [15]. Both PfHsp70-z and PfHsp70-1 are thought to play a crucial role under the physiologically stressful conditions the parasites encounter during their life cycle. PfHsp70-1 and PfHsp70-z are particularly important at the blood stage during the development of clinical malaria [16,17].

Pterocarpus angolensis DC belongs to the family of Fabaceae [18]. P. angolensis has been reported to have antimicrobial properties and is used to treat malaria, among other diseases [18-21]. Ziziphus mucronata belongs to the Rhamnaceae family and is found in most parts of South Africa [22]. Stem and bark infusions have previously been reported to have antimicrobial activity [23,24].

Heat shock proteins have been proposed as antimalarial drug targets $[9,11]$ and thus elucidating their inhibitors presents an alternative option towards antimalarial drug discovery. We recently demonstrated inhibition of parasite Hsp70 function by the cyclic peptide antibiotic, polymyxin B [14]. As plants contain a wide range of potential small molecule inhibitors of proteins, we speculated that some of the inhibitors may target heat shock protein function.

We investigated the effects of the P. angolensis and Z. mucronata extracts on the functional features of both PfHsp70-1 and PfHsp70-z. In addition, we investigated the effects of the P. angolensis and 
Z. mucronata extracts on the viability of P. falciparum 3D7 parasites maintained at the blood stage. Data from this study demonstrate that select fractions from the P. angolensis and Z. mucronata extracts inhibit Hsp70 chaperone function. Furthermore, our findings established that the P. angolensis and Z. mucronata fractions possess antiplasmodial activity. We discuss the implications of our findings and the prospects of further characterizing the compounds in P. angolensis and Z. mucronata extract fractions as inhibitors of Hsp70 function in malaria.

\section{Results}

\subsection{Z. mucronata and P. angolensis Extracts Contain Phenolic Compounds}

We quantified the phenolic compounds in the P. angolensis and Z. mucronata fractions using mass spectrometric (MS) analysis (Supplementary Table S1). The MS analysis showed that ZF2 contains the highest phenolic content (Table 1). In addition, both P. angolensis and Z. mucronata fractions contain epicatechin, and low levels of gallic acid, taxifolin and rutin (Table 1).

Table 1. Quantification of phenolic compounds in Z. mucronata and P. angolensis extracts.

\begin{tabular}{|c|c|c|c|c|c|c|c|}
\hline \multirow{2}{*}{ Samples } & \multicolumn{7}{|c|}{ Compounds [mg/g Dry Weight]/( \pm Standard Deviation; $n=3)$} \\
\hline & Protocatechin & Catechin & Gallic Acid & Caffeic Acid & Rutin & Epicatechin & Taxifolin \\
\hline ZF1 & ND & - & - & - & - & - & - \\
\hline ZF3 & $0.26( \pm 0.02)$ & $1.71( \pm 0.21)$ & - & $2.72( \pm 0.20)$ & - & $4.06( \pm 0.30)$ & $0.09( \pm 0.01)$ \\
\hline ZF4 & $0.46( \pm 0.03)$ & $0.72( \pm 0.16)$ & $0.41( \pm 0.03)$ & - & - & $1.47( \pm 0.02)$ & $0.04( \pm 0.002)$ \\
\hline ZF5 & $0.58( \pm 0.04)$ & $0.23( \pm 0.04)$ & $0.18( \pm 0.02)$ & - & - & $0.41( \pm 0.01)$ & $0.01( \pm 0.001)$ \\
\hline $\mathrm{PaF} 2 \mathrm{~b}$ & ND & - & & - & - & $0.19( \pm 0.001)$ & - \\
\hline PaF3a & ND & - & - & - & - & $0.02( \pm 0.003)$ & - \\
\hline PaF3b & ND & - & - & - & - & $0.41( \pm 0.006)$ & - \\
\hline PaF4 & ND & - & - & - & - & $0.05( \pm 0.0001)$ & - \\
\hline
\end{tabular}

Legend: ND-not detected; Standard deviations are for intraday variation.

\subsection{Z. mucronata and P. angolensis Extracts Suppress the Chaperone Function of Both PfHsp70-1 and PfHsp $70-z$}

We expressed and purified both recombinant PfHsp70-1 and PfHsp70-z from E. coli XL1 Blue and E. coli JM109 cell lines, respectively (Supplementary Figure S1). Using the recombinant forms of both PfHsp70-1 and PfHsp70-z, we previously showed that they are heat stable and are capable of suppressing the heat induced aggregation of model substrates such as MDH [10,13,14,25]. In a previous study, we observed that the chaperone activity (suppression of heat induced protein aggregation) of PfHsp70-z was not influenced by nucleotides while ATP inhibited the chaperone activity of PfHsp70-1, respectively [10,13]. In the current study, $\mathrm{MDH}$ was subjected to heat stress at $48{ }^{\circ} \mathrm{C}$, and as expected it did aggregate in the absence of chaperones (Table 2).

Table 2. Comparative thermal stability of PfHsp70-1, PfHsp70-z and MDH in the presence of P. angolensis and Z. mucronata extracts.

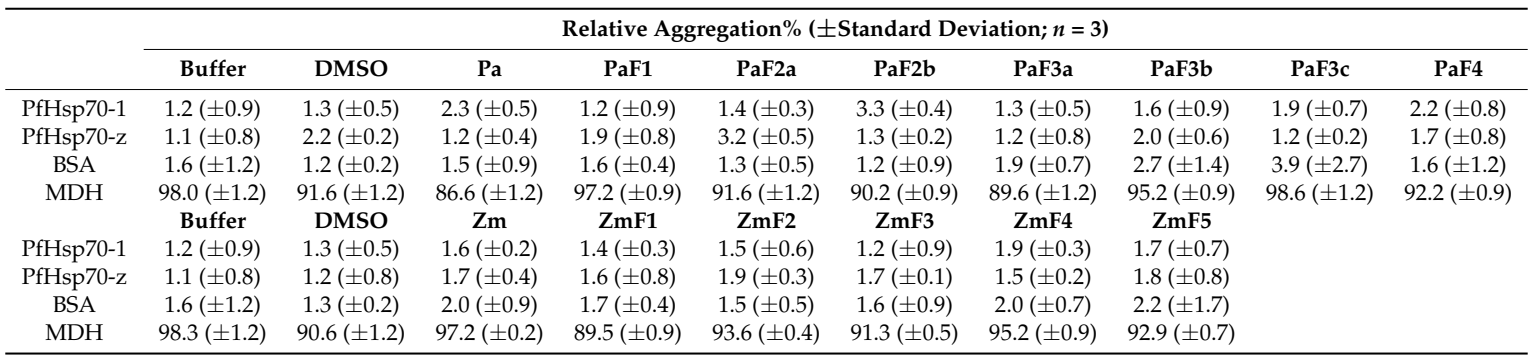


In the presence of a non-chaperone protein (BSA), $\mathrm{MDH}$ also aggregated in response to heat stress. It was important to validate that the solvent used $0.01 \%$ DMSO did not affect the stability for the chaperones, and for this reason it was used as baseline control. When the chaperones were excluded from the reaction mixture, only $\mathrm{MDH}$ fully aggregated in the presence and absence

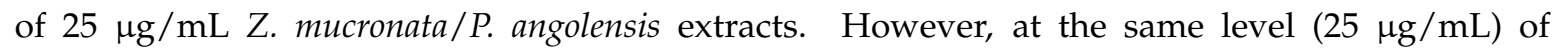
Z. mucronata/P. angolensis extracts did not influence the solubility of either PfHsp70-1 or PfHsp70-Z subjected to heat stress. Assessment of the thermal stability of PfHsp70-1/PfHsp70-z/BSA/MDH was conducted by monitoring the heat induced aggregation of the respective protein in vitro at $48^{\circ} \mathrm{C}$. The degree of aggregation was estimated by monitoring the increase in optical density using spectroscopy at $320 \mathrm{~nm}$. Relative aggregation was normalized to spontaneous MDH aggregation. Standard deviations obtained from three replicate assays are shown.

In the presence of $P$. angolensis crude extract, $\mathrm{Pa}$ and the fractions $\mathrm{PaF} 1$ and $\mathrm{PaF} 4$, the chaperone activities of either PfHsp70-1 or PfHsp70-z were inhibited in a concentration dependent manner (Figure 1, Table 3). However, the P. angolensis fractions $(\mathrm{PaF} 2 \mathrm{a} / \mathrm{b} ; \mathrm{PaF} 3 \mathrm{a} / \mathrm{b} / \mathrm{c})$ did not suppress the activities of the two proteins (Figure 1).

A
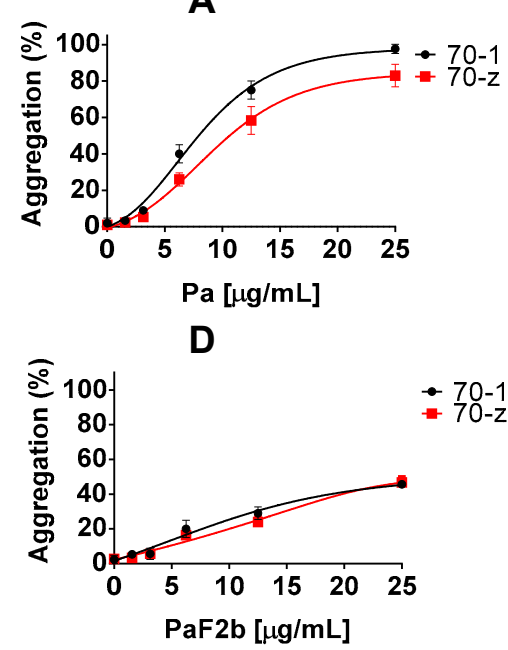

G

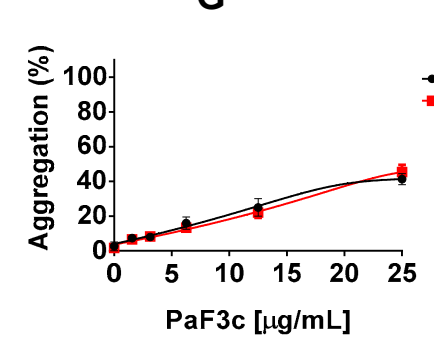

B

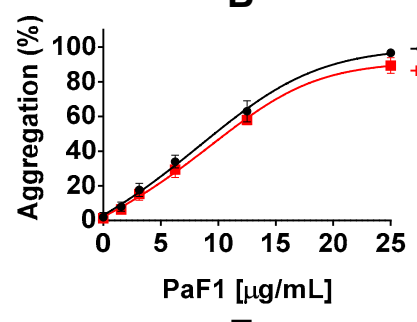

E

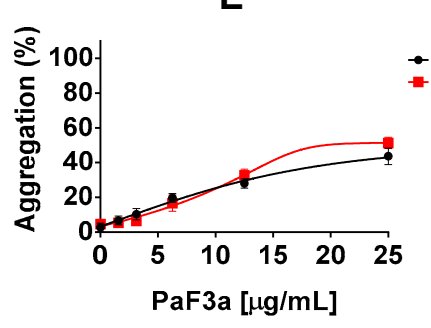

H

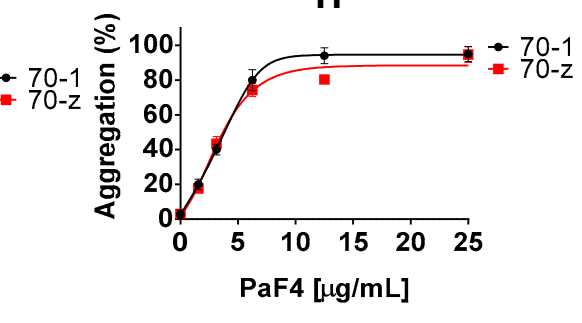

C

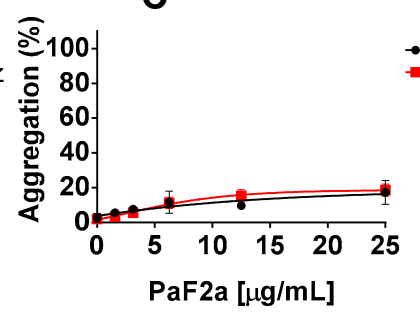

F

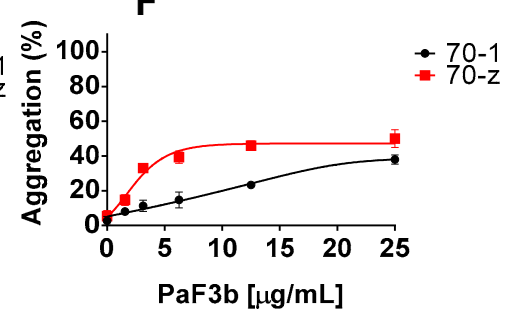

Figure 1. P. angolensis extracts suppress chaperone function of PfHsp70-1 and PfHsp70-z. The chaperone function of PfHsp70-1 and PfHsp70-z was investigated by monitoring the heat induced aggregation of $\mathrm{MDH}$ in the presence of PfHsp70-1/PfHsp70-z (chaperone) in vitro at $48{ }^{\circ} \mathrm{C}$ for $60 \mathrm{~min}$. The resultant heat induced aggregation of $\mathrm{MDH}$ was estimated by taking readings at $360 \mathrm{~nm}$. The values were normalized to the aggregation of spontaneous $\mathrm{MDH}$ aggregation in the absence of PfHsp70-1 (70-1)/PfHsp70-z (70-z). The assay was repeated in the presence of the various plant extracts and represented by various panels: (A) Pa, (B) PaF1; (C) PaF2a; (D) PaF2b; (E) PaF3a; (F) PaF3b; (G) PaF3c; (H) PaF4. Standard deviations obtained from three replicate assays are shown. 
Table 3. Comparative $\mathrm{IC}_{50}$ inhibition of P. angolensis and Z. mucronata extracts on PfHsp70-1 and PfHsp70-z chaperone activities.

\begin{tabular}{ccccccccccc}
\hline & \multicolumn{10}{c}{ IC $_{50}(\boldsymbol{\mu} \mathbf{g} / \mathbf{m L}) /[ \pm$ Standard Deviation] } \\
\cline { 2 - 11 } & Pa & PaF1 & PaF2a & PaF2b & PaF3a & PaF4 & Zm & ZmF2 & ZmF4 & ZmF5 \\
\hline PfHsp70-1 & $12.3( \pm 1.2)$ & $0.5( \pm 0.2)$ & ND & $15.6( \pm 2.1)$ & $10.1( \pm 1.5)$ & $0.8( \pm 0.3)$ & $9.3( \pm 1.8)$ & $3.1( \pm 1.1)$ & ND $( \pm 5.2)$ & $6.0( \pm 1.2)$ \\
PfHsp70-z & $17.5( \pm 2.1)$ & $9.0( \pm 1.1)$ & $6.5( \pm 0.2)$ & ND & $13.7( \pm 2.0)$ & $6.1( \pm 1.0)$ & $13.8( \pm 2.1)$ & $4.3( \pm 0.4)$ & $3.2( \pm 1.0)$ & $6.4( \pm 0.3)$ \\
\hline
\end{tabular}

ND—undetermined ( $>25 \mu \mathrm{g} / \mathrm{mL})$, PaF3b, PaF3c, ZF1 and ZF3 were not tested. Standard deviations obtained from three replicate assays are shown.

Similarly, the crude extract of Z. mucronata $(\mathrm{Zm})$ and fractions $\mathrm{ZmF} 2, \mathrm{ZmF} 4$ as well as $\mathrm{ZmF5}$ suppressed the chaperone function of both PfHsp70-1 and PfHsp70-z in a concentration dependent manner (Figure 2, Table 3). However, fractions $\mathrm{ZmF1}$ and $\mathrm{ZmF3}$ were not effective at suppressing the chaperone function of both proteins.

A

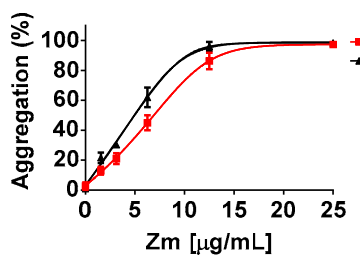

D

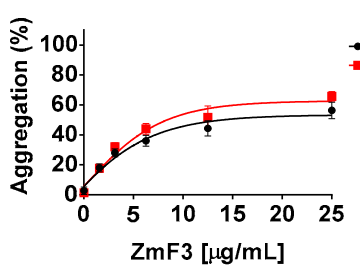

B

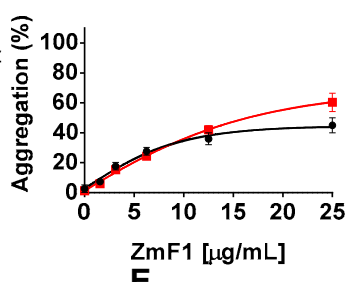

E
C

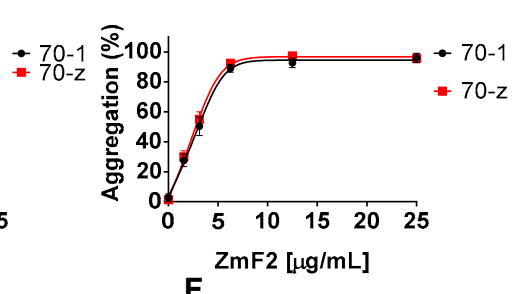

$\mathbf{F}$

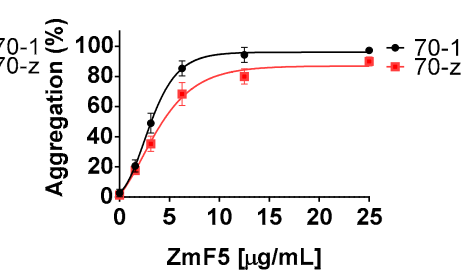

Figure 2. Inhibition of the chaperone activities of PfHsp70-1 and PfHsp70-z by Z. mucronata extracts. Heat induced aggregation of MDH was monitored in the presence and absence of PfHsp70-1/PfHsp70-z $(70-1 / 70-\mathrm{z})$ at $48^{\circ} \mathrm{C}$ for $60 \mathrm{~min}$. The resultant aggregates were estimated at $360 \mathrm{~nm}$. The values were normalized to aggregation of spontaneous MDH aggregation in the absence of PfHsp70-1/PfHsp70-z. Data obtained in the presence of various plant extracts is provided: (A) Zm; (B) ZmF1; (C) ZmF2; (D) ZmF3; (E) ZmF4; (F) ZmF5. Standard deviations obtained from three replicate assays are shown.

\subsection{Select P. angolensis and Z. mucronata Extracts Inhibit PfHsp70-1 and PfHsp70-z ATPase Activity}

Previous studies have shown that PfHsp70-z and PfHsp70-1 both bind to ATP with comparable affinities. Furthermore, both proteins exhibit ATPase activities within the same range of magnitude [13]. In the current study, the ATPase activities of both Hsp70s were determined under ATP saturating levels $(5 \mathrm{mM})$, while the concentration of P. angolensis was varied $(0-25 \mu \mathrm{g} / \mathrm{mL})$ (Figure 3$)$. We noted that the ATPase activities of the respective protein decreased with increase in the concentration of fractions Pa, PaF1, PaF4 (Figure 3). This suggests that the fractions contain compounds that specifically interfered with the ATPase function of both PfHsp70-1 and PfHsp70-z. On the other hand, fractions $\mathrm{PaF} 2 \mathrm{a} / \mathrm{b}, \mathrm{PaF} 3 \mathrm{a} / \mathrm{b} / \mathrm{c}$, were generally ineffective (Figure 3).

The ATPase assay was repeated in the presence of varying amounts (up to a maximum of $25 \mu \mathrm{g} / \mathrm{mL}$ ) of the Z. mucronata extract (Figure 4). As the concentration of crude extract $\mathrm{Zm}$ and fractions $\mathrm{ZmF}$, ZmF4, ZmF5 increased, the ATPase activities of the two proteins were suppressed. This again suggests that these fractions contain compounds that inhibit the ATPase activity of Hsp70. On the other hand, fractions $\mathrm{ZmF1}$ and $\mathrm{ZmF3}$ did suppress the ATPase activities of the two proteins, albeit less effectively (Figure 4). 


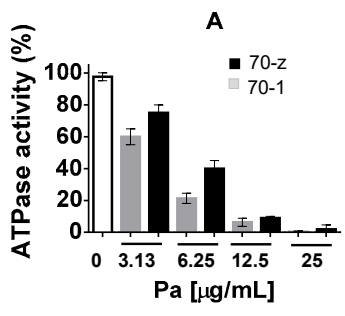

D

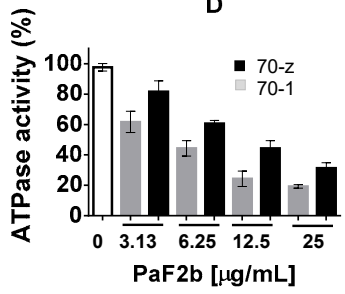

G

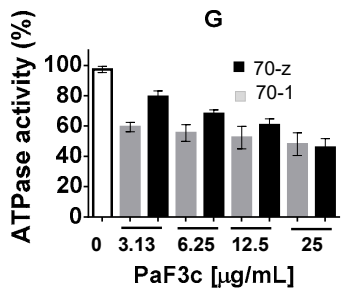

B

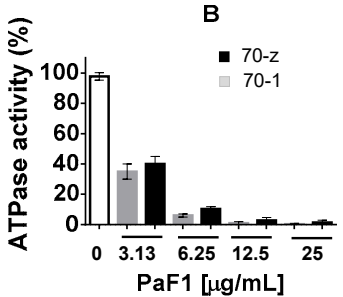

E

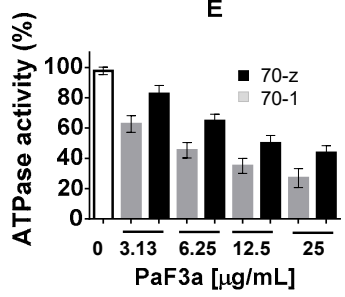

$\mathrm{H}$

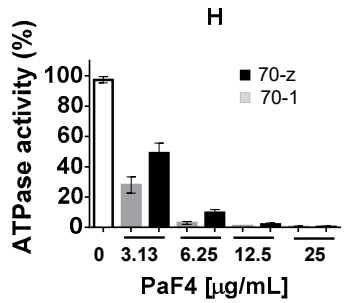

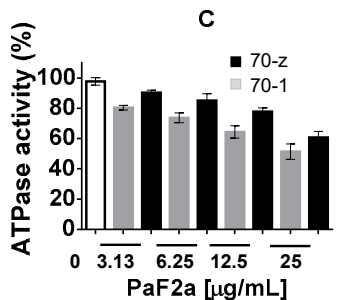

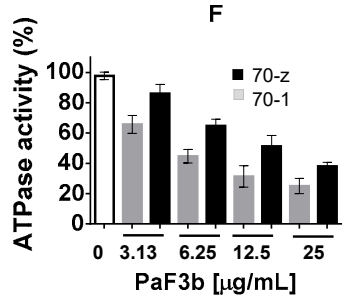

Figure 3. P. angolensis extracts inhibit the basal ATPase activity of PfHsp70-1 and PfHsp70-z. The basal ATPase activities of PfHsp70-1 (70-1) and PfHsp70-z (70-z) were analysed in the presence of various plant extracts. The values were normalized to the basal ATPase activities of each protein obtained in the absence of plant extract. The effects of plant extract under variable concentrations were then investigated and represented: (A) Pa; (B) PaF1; (C) PaF2a; (D) PaF2b; (E) PaF3a; (F) PaF3b; (G) PaF3c; (H) PaF4. Standard deviations obtained from three replicate assays are shown.
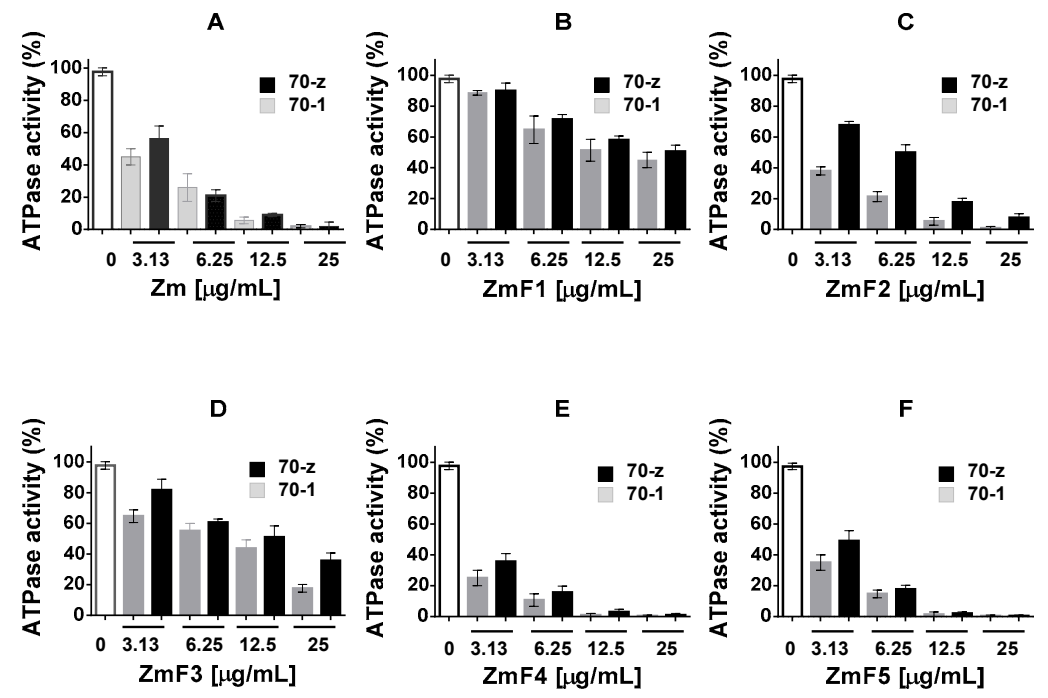

Figure 4. Inhibition of the basal ATPase activity of PfHsp70-1 and PfHsp70-z by Z. mucronata extracts. The basal ATPase of PfHsp70-1 (70-1)/PfHsp70-z (70-z) were analysed in the presence of increasing amounts of the various plant extracts. The values were normalized to basal ATPase of each protein obtained in the absence of plant extract. The effects of the variable concentrations of plant extracts were then investigated and presented: (A) Zm; (B) ZmF1; (C) ZmF2; (D) ZmF3; (E) ZmF4; (F) ZmF5. Standard deviations obtained from three replicate assays are shown. 


\subsection{Z. mucronata and P. angolensis Extracts Inhibit Growth of Plasmodium falciparum Parasites}

Growth inhibition assays were conducted on P. falciparum 3D7 maintained at the blood stage based on the P. falciparum lactate dehydrogenase (pLDH) method as previously described [26]. First it was important to validate the antimicrobial sensitivity of the parasites. For this reason, $1 \mu \mathrm{M}$ chloroquine, a known antimalarial drug that inhibits P. falciparum 3D7 growth [27], was used as a positive control. Furthermore, DMSO was used within similar range $(0.05 \%)$ as previously recommended to allow maximum parasite viability [28]. P. falciparum 3D7 cells cultured in the absence of extracts were used as negative controls, and these as expected exhibited maximum viability. The treatment of parasite cultures with $25 \mu \mathrm{g} / \mathrm{mL}$ of $P$. angolensis/Z. mucronata extracts resulted in the inhibition of parasite growth. The crude extracts $\mathrm{Pa}, \mathrm{Zm}$ as well as fractions PaF1, PaF4, ZmF2 and ZmF5 inhibited parasite growth by more than $75 \%$. In addition, the fractions that inhibited $75 \%$ of parasite growth were further analysed to determine the concentration that suppressed parasite growth by up to $50 \%$ (IC 50 ). As a limitation, for some extracts the concentration range used was not high enough to obtain $0 \%$ viability. P. falciparum 3D7 cell line is a known chloroquine sensitive strain and in the current study chloroquine registered an $\mathrm{IC}_{50}$ of $8.5 \mathrm{ng} / \mathrm{mL}$ which is within the reported range from previous studies (Table 4, [27]). We observed that P. angolensis fractions 1 and 4 both exhibited significantly lower $\mathrm{IC}_{50}$ values in comparison with $Z$. mucronata fractions 2 and $5(p<0.05$, Table 4$)$. This suggests that P. angolensis contain compounds with more potent antiplasmodial properties than Z. mucronata.

Table 4. $\mathrm{IC}_{50}$ values for the antiplasmodial activities of P. angolensis and Z. mucronata extracts

\begin{tabular}{cc}
\hline Compound & IC $_{\mathbf{5 0}}(\boldsymbol{\mu g} / \mathbf{m L}) /[ \pm$ Standard Deviation; $\boldsymbol{n}=\mathbf{2}]$ \\
\hline $\mathrm{Pa}$ & $13.87( \pm 0.20)$ \\
$\mathrm{PaF} 1$ & $0.7945( \pm 0.002)$ \\
$\mathrm{PaF} 4$ & $1.961( \pm 0.01)$ \\
$\mathrm{Zm}$ & $7.4( \pm 0.3)$ \\
$\mathrm{ZmF} 2$ & $6.404( \pm 0.02)$ \\
ZmF5 & $19.9( \pm 0.3)$ \\
Chloroquine & $0.008522( \pm 0.0004)$ \\
\hline
\end{tabular}

\section{Discussion}

The emergence of multidrug resistance by P. falciparum parasites has resulted in a high unmet need for novel antimalarial drugs to combat malaria. This study provides the first direct evidence of the anti-chaperone function and antiplasmodial activity of extracts from both Z. mucronata and P. angolensis plants. Our findings suggest that the two plants contain compounds that inhibit parasite growth in vitro. In addition, some of the plant compounds have the capability to suppress the ATPase and chaperone functions of two prominent P. falciparum chaperones, PfHsp70-1 and PfHsp70-z which are localized to the parasite cytosol [13]. We previously observed that compounds that inhibit PfHsp70-z and PfHsp70-1 such as polymyxin B [14] and (-)-epigallocatechin-3-gallate (EGCG, unpublished data) also inhibit parasite growth. In addition, such small molecule inhibitors also abrogated association of the two chaperones with their functional partners [14]. It is therefore possible that both Z. mucronata and P. angolensis plants contain compounds that interfere with either ATP binding by Hsp70 as well those that interfere with capacity of the chaperone to bind substrates. Although the fractions derived here exhibited higher $\mathrm{IC}_{50}$ than chloroquine, they showed promising antimalarial activity. It is also possible that the plant extracts may have inhibited other functions besides the protein folding pathway of the parasite, in exerting their antiplasmodial activity.

The plant extracts used in the current study were fractioned using increasing polarity of the respective solvents. It is well known that different compounds are fractioned under various solvents polarity. For example, it has been previously reported that hexane generated fractions lack antimalarial activity [29-31], which is in line with the current results as ZmF1 which lack phenolic compounds, 
did not exhibit significant anti-chaperone nor anti- malarial activities, respectively (Figures 2 and 4 , Tables 1 and 2).

Hexane fractions have been shown to comprise phenols, triterpenoids, terpenoids and tannins $[29,31]$. Interestingly, results from this study showed that fraction PaF1 (50\% ethyl acetate and 50\% hexane) exhibited potent antiplasmodial activity (Table 3). This suggests that despite the hexane derived ZmF1 fraction lacking antimalarial activity, increasing the ethyl acetate component to 50\% (fraction PaF1) led to the recovery of compounds that do possess antimalarial activity. However, the antiplasmodial activity was not only a function of the solvent used to extract the products, but was also dependent on the plant source (Table 3).

Ethyl acetate fractions generally contain phenols, triterpenoids, terpenoids, flavonoids and steroids [30,31]. Of these, flavonoids and phenols have been previously reported to possess potent antimalarial activity [29,31]. This composition possibly explains the antiplasmodial activity observed in this study. Methanol extracts have been previously reported to contain a variety of compounds [32]. It would therefore be important to establish the compounds contained in fractions ZmF5 and PaF4 that exhibit antiplasmodial activity.

One of the main pathways targeted by most antimalarial compounds is the oxido-reductory system [33,34]. In the current study, we explored the protein folding pathway as a potential antiplasmodial drug target. This is because heat shock proteins have been proposed as prospective antimalarial drug targets [3,9]. However, heat shock proteins tend to be conserved, thereby confounding their suitability as antimalarial drug targets. One of the validated targets of the extracts derived in the current study is the essential, cytosol localized parasite heat shock protein, PfHsp70-z [8,13]. The PfHsp70-z belongs to Hsp110 subfamily which generally exhibit very low sequence conservation compared to its human equivalence [13]. Hence, the observed inhibition of PfHsp70-z by the compounds isolated from this study is important towards antiplasmodial drug design. In addition, P. falciparum proteome is estimated to be comprised of asparagine repeat proteins which constitute at least $25 \%$ of the population asparagine repeat proteins are generally aggregation prone. The PfHsp70-z has been reported to possess independent chaperone function and the protein is deemed to play an important function in regulating protein quality control, especially buffering aggregation prone proteins of the parasite against cellular stress [13].

Polyphenols were generally present in all the fractions irrespective of the extraction solvents used, except fraction PaF2a which exhibited significantly lower polyphenol content (Table 1) [35]. Previous studies have shown that flavonoids are extracted effectively using ethyl acetate [29]. The PaF1 was the only fraction with a $\mathrm{C}=\mathrm{O}$ in the IR region of phenolic esters. This could represent saponins (i.e., terpenoids with glycosides) or flavonoids with glycosides. The PaF1 did not display any antioxidant activities and further analysis using MS confirmed that it lacks the phenolic compounds [35]. However, the presence of the alkyl group in the IR data could signify the presence of terpenoids which is consistent with the potent antiplasmodial activity that it exhibited (Table 4) [35]. In addition, extracts F2 and F4 from Z. mucronata obtained using a solvent rich in ethyl acetate showed strong antiplasmodial activity (Table 1). This observation is in line with previous studies which demonstrated that green tea plant extract and husk extract (fractions of Zea mays) obtained using ethyl acetate exhibited notable antiplasmodial function $[29,31]$.

Altogether, the current study provides the first evidence for the antiplasmodial activity for P. angolensis and Z. mucronata stem bark extractions. In addition, some of the obtained fractions inhibited the chaperone functions of two cytosol localized parasite Hsp70 homologues. The findings suggest that amongst the compounds present in the extracts some target Hsp70 function. For example, notable in some of the fractions are catechins whose antimalarial activity has also been reported [29]. We previously showed that compounds that inhibit parasite Hsp70, tend to not only inhibit the chaperone function of the protein but abrogate association of Hsp70 with its functional associates [14]. This makes inhibition of parasite Hsp70 an attractive drug target. There is need to further characterize the fractions obtained here in order to identify compounds that are responsible for the observed 
antiplasmodial activity towards expanding the drug arsenal against malaria. This is especially important in the wake of drug resistance being increasingly mounted by malaria parasites.

\section{Materials and Methods}

\subsection{Materials}

Unless, specified chemical reagents used in this study were purchased from Merck (Darmstadt, Germany), Sigma Aldrich (St. Louis, MO, USA), and Melford (Suffolk, UK).

\subsection{Fractionation of Pterocarpus angolensis and Ziziphus mucronata Stem Bark Extracts}

The plants were collected by Muendi Sigidi in the Vuwani area (Hamangilasi, Vhembe District Municipality of Limpopo Province, South Africa) on the 28th of January 2015. The voucher specimen number for Pterocarpus angolensis is MPT00118. The voucher specimen number for Ziziphus mucronata is MPT00123 and the plant was collected at Mukomawabani (Tshikondeni, Vhembe District Municipality) in 2014. The two plants were identified using their Venda vernacular names, Makhalu and Mutondo in their natural habitat in the Vhembe District of Limpopo Province. The plants were later confirmed by the taxonomic rank at the Department of Botany, University of Venda with reference to the international plant name index (Ziziphus mucronata Willd [MPT00123] and Pterocarpus angolensis DC [MPT00118] as previously described [21]. Stem bark samples were processed for each plant, the samples were first air dried and ground to powder using a Büchi mixer (Büchi Labortechnik AG, Flawil, Switzerland). The samples of $P$. angolensis bark were soaked as $5 \%(w / v)$ to solvent $1(50 \%$ dichloromethane and $50 \%$ methanol) at room temperature for $24 \mathrm{~h}$. Then, the extract was filtered and evaporated with a rotor vapor at $40{ }^{\circ} \mathrm{C}$ to obtain the crude extract $(\mathrm{Pa})$. The crude extract was fractioned using a silica gel 60 column chromatography and was eluted with hexane and increasing polarity with ethyl acetate and finally methanol to obtain 7 fractions as previously described [35]. Briefly, P. angolensis fraction 1 (PaF1) was obtained from 50\% hexane, 50\% ethyl acetate (1:1), PaF2a and $2 \mathrm{~b}$ were obtained from $90 \%$ ethyl acetate, $10 \%$ methanol; PaF3a-c were obtained from $70 \%$ ethyl acetate and $30 \%$ methanol while PaF4 was obtained from 10\% ethyl acetate 90\% methanol [35]. The stem bark for Ziziphus mucronata (MPT00123) was also processed as previously described [21] and used to extract fractions by soaking in ethanol to obtain the crude extract $(\mathrm{Zm})$. The $\mathrm{Zm}$ was also subjected to silica gel column chromatography and eluted in five fractions. Z. mucronata fraction $1,(\mathrm{ZmF1})$ was eluted with $70 \%$ hexane and $30 \%$ ethyl acetate; fraction $2(\mathrm{ZmF} 2)$ and fraction $3(\mathrm{ZmF} 3)$ were eluted with $90 \%$ ethyl acetate and $10 \%$ methanol; fraction $4(\mathrm{ZmF} 4)$ was eluted with $70 \%$ ethyl acetate and $30 \%$ methanol and fraction 5 (ZmF5) was eluted with 30\% ethyl acetate and 70\% methanol.

\subsection{Quantification of Phenolics in Pterocarpus angolensis and Ziziphus mucronata Extracts}

The presence of caffeic acid, catechin, epicatechin, gallic acid, protocatechuic acid, taxifolin and rutin was determined in the fractions of P. angolensis and Z. mucronata using a UPLC-QToF-MS method developed by the Central Analytical Facilities (CAF), University of Stellenbosh, South Africa. The method was validated for linearity and precision using standard procedures [36,37]. A concentration range of $1-100 \mu \mathrm{g} / \mathrm{mL}$ was used to construct the calibration curves for, caffeic acid, gallic acid, protocatechuic acid, and taxifolin, while a concentration range of $3.5-111 \mu \mathrm{g} / \mathrm{mL}$ was used to construct calibration curves for catechin, epicatechin, and rutin, respectively. The phenolics were identified and quantified by comparing the retention time of the standards with the samples analysed (Supplementary Table S1). A Synapt G2 quadrupole time-of-flight mass spectrometer (Waters, NewYork, NY, USA) was used for LC-MS analysis. It was fitted with a Waters Ultra pressure liquid chromatograph and photo diode array detection. Separation was achieved on a Waters BEH C18, $2.1 \times 100 \mathrm{~mm}$ column with $1.7 \mu \mathrm{m}$ particles. A gradient was applied using $0.1 \%$ formic acid (solvent A) and acetonitrile containing $0.1 \%$ formic acid (solvent $\mathrm{B}$ ). The gradient started at $100 \%$ solvent $\mathrm{A}$ for 1 minute and changed to $28 \%$ B over $22 \mathrm{~min}$ in a linear way. It then went to $40 \%$ B over $50 \mathrm{~s}$ and a 
wash step of $1.5 \mathrm{~min}$ at $100 \% \mathrm{~B}$, followed by re-equilibration to initial conditions for $4 \mathrm{~min}$. The flow rate was $0.3 \mathrm{~mL} / \mathrm{min}$ and the column was kept at $55^{\circ} \mathrm{C}$. The injection volume was $2 \mu \mathrm{L}$. Data was acquired in MSE mode which consisted of a low collision energy scan (6V) from $m / z 150$ to 1500 and a high collision energy scan from $m / z 40$ to 1500 . The high collision energy scan was done using a collision energy ramp of 30-60 V. The photo diode array detector was set to scan from 220 to $600 \mathrm{~nm}$. The mass spectrometer was optimized for best sensitivity, a cone voltage of $15 \mathrm{~V}$, desolvation gas was nitrogen at $650 \mathrm{~L} / \mathrm{h}$ and desolvation temperature $275^{\circ} \mathrm{C}$. The instrument was operated with an electrospray ionization probe in the negative mode. Sodium formate was used for calibration and leucine encephalin was infused in the background as lock mass for accurate mass determinations.

\subsection{Expression and Purification of Recombinant Proteins}

A construct expressing PfHsp70-1 (pQE30/PfHsp70-1 [10], and PfHsp70-z pQE30/PfHsp70-z [11]) were used for the expression of recombinant PfHsp70-1 and PfHsp70-z proteins using E. coli XL1 Blue and JM109 cells respectively, following a previously described method [10,11]. The recombinant proteins were purified using affinity chromatography as previously described [13].

4.5. Analysis of the Effect of P. angolensis and Z. mucronata Extracts on the Chaperone Function of PfHsp70-1 and PfHsp70-z

The effects of P. angolensis and Z. mucronata extracts on the chaperone function of both PfHsp70-1 and PfHsp70-z were investigated by monitoring the heat-induced aggregation of a model protein, malate dehydrogenase (MDH) from Photinus pyralis (Sigma-Aldrich, Saint Louis, MO, USA) as previously described [13] with minor modifications. In order to determine the effects of different plant extracts on the chaperone function of both PfHsp70-1 and PfHsp70-Z, the assay was conducted in the presence of varying amounts of the plant extract $(0-50 \mu \mathrm{g} / \mathrm{mL})$. The aggregation reaction was monitored at $320 \mathrm{~nm}$ for $1 \mathrm{~h}$ and data was processed using GraphPad prism 6 (San Diego, CA, USA).

\subsection{Investigation of the Effects of P. angolensis and Z. mucronata Extracts on Basal Hsp70 ATPase Activity}

The basal ATPase activities of PfHsp70-1 and PfHsp70-z Hsp70 were determined by a colorimetric method as previously described $[13,14]$. In order to determine the effects of P. angolensis and Z. mucronata extracts on the basal ATPase activity on parasite Hsp70s, the assay was repeated in the presence of varying amounts $(0.1-50 \mu \mathrm{g} / \mathrm{mL})$ of either P. angolensis and Z. mucronata extract. As controls, the respective boiled Hsp70 protein was used to cater for spontaneous hydrolysis of ATP.

\subsection{Investigation of the Effects of P. angolensis and Z. mucronata Extracts on P. falciparum Parasite Growth}

P. falciparum 3D7 parasites were grown and maintained in continuous culture as previously described $[12,38]$. The $P$. falciparum lactate dehydrogenase (pLDH) method was used to monitor the growth of $P$. falciparum 3D7-infected erythrocytes subjected to different amounts of $(0.01-25 \mu \mathrm{g} / \mathrm{mL})$ $P$. angolensis and Z. mucronata extracts treatments as previously described [26]. Initial growth assay screening was conducted at $25 \mu \mathrm{g} / \mathrm{mL}$ plant extract concentration. The plant extracts with $75 \%$ growth inhibition of parasite were further analysed for $\mathrm{IC}_{50}$ determination by titrating (range $0.001-20 \mu \mathrm{g} / \mathrm{mL}$ ) the plant extract concentration. Analysis of the pLDH data for growth inhibition assay was carried out using GraphPad Prism 6. All experiments in this study were repeated at least three times with three runs each time.

\section{Conclusions}

We demonstrated that extracts of both P. angolensis and Z. mucronata exhibit antiplasmodial activity. Crude extracts of the two plants and some of their respect fractions (PaF1, PaF4, ZmF2, ZmF4 and $\mathrm{ZmF5}$ ) effectively suppressed the chaperone activities and ATPase functions of the two parasite cytosol localized chaperones (PfHsp70-1 and PfHsp70-z). This suggests that the plants contain compounds that may abrogate ATP binding by Hsp70, and at the same time, the compounds interfere with the 
capacity of the chaperones to bind their substrates (misfolded proteins). Furthermore, that fractions, PaF1, PaF4, ZmF2, and ZmF5 also further inhibited parasite growth provides evidence that the plant products abrogate Hsp70 function to inhibit parasite growth. However, it cannot be excluded that some of the compounds from the plants may target other pathways in the parasite. It is important to further establish the exact compounds implicated in the processes leading to parasite death.

Supplementary Materials: The following are available online; Figure S1: Expression purification of recombinant PfHsp70-1 and PfHsp70-z; Table S1: The linear range, correlation coefficient, LOD and LOQ of each standard used in quantification of phenolics.

Acknowledgments: This project was supported through a grant (L1/402/14-1) provided to AS by the Deutsche Forchungsgemeinshaft (DFG) under the theme, "German-African Cooperation Projects in Infectiology" and the Research and Innovation Directorate (S/15/MBY10) grants provided by the University of Venda, to ANT. The research was coordinated by the Office of the Dean, School of Mathematical and Natural Sciences, University of Venda as part of an interdisciplinary research and capacity building project of the School. We are grateful to the Department of Science and Technology/National Research Foundation (NRF) of South Africa for providing an equipment grant (UID, 75464) and NRF mobility grant (UID, 92598) awarded to AS, South African Medical Research Council (MRC) with funds from National Treasury under its Economic Competitiveness and Support Package, and Rhodes University Sandisa Imbewu (awarded to HH). TZ is a recipient of the Claude Leon Post-Doctoral fellowship and African-German Network of Excellence in Science junior researcher grant; AS is a recipient of a Georg Foster research fellowship awarded by the Alexander von Humboldt Foundation, Germany.

Author Contributions: T.Z. and A.S. conceived and designed experiments; interpreted the data, and wrote the manuscript; T.Z. conducted experiments; A.S. contributed reagents; H.H. analysed data, contributed reagents; C.P.A. and I.I.D.R. fractionated the crude extracts, quantified the phenolics in the fractions; C.P.A. wrote the manuscript; M.P.T. interpreted data; M.T.S. and A.N.T. provided reagents; N.P. conceptualized the interdisciplinary project team, assisted in obtaining the Univen grant and editing the manuscript. All authors discussed the contents of the manuscript and approved the submission.

Conflicts of Interest: The authors declare no conflict of interest. The founding sponsors had no role in the design of the study; in the collection, analyses, or interpretation of data; in the writing of the manuscript, and in the decision to publish the results.

\section{References}

1. World Health Organization. World Malaria Report. Available online: http://www.who.int/malaria/ publications/world-malaria-report-2016/report/en/ (accessed on 27 May 2017).

2. Hartl, F.U. Heat shock proteins in protein folding and membrane translocation. Semin. Immunol. 1991, 3, 5-16. [PubMed]

3. Zininga, T.; Shonhai, A. Are heat shock proteins druggable candidates? Am. J. Biochem. Biotechnol. 2014, 10, 211-213. [CrossRef]

4. Shonhai, A. Role of Hsp70s in development and pathogenicity of plasmodium species. In Heat Shock Proteins of Malaria; Shonhai, A., Blatch, G., Eds.; Springer: New York, NY, USA, 2014; Volume 1, pp. 47-70. [CrossRef]

5. Kampinga, H.H.; Craig, E. The Hsp70 chaperone machinery: J proteins as drivers of functional specificity. Nat. Rev. Mol. Cell Biol. 2010, 11, 579-592. [CrossRef] [PubMed]

6. Hennessy, F.; Nicoll, W.S.; Zimmermann, R.; Cheetham, M.E.; Blatch, G.L. Not all J domains are created equal: Implications for the specificity of Hsp40-Hsp70 interactions. Protein Sci. 2005, 14, 1697-1709. [CrossRef] [PubMed]

7. Dragovic, Z.; Broadley, S.A.; Shomura, Y.; Bracher, A.; Hartl, F.U. Molecular chaperones of the Hsp110 family act as nucleotide exchange factors of Hsp70s. EMBO J. 2006, 25, 2519-2528. [CrossRef] [PubMed]

8. Shonhai, A.; Boshoff, A.; Blatch, G.L. The structural and functional diversity of Hsp70 proteins from Plasmodium falciparum. Protein Sci. 2007, 16, 1803-1818. [CrossRef] [PubMed]

9. Shonhai, A. Plasmodial heat shock proteins: Targets for chemotherapy. FEMS Immunol. Med. Microbiol. 2010, 58, 61-74. [CrossRef] [PubMed]

10. Shonhai, A.; Botha, M.; de Beer, T.A.P.; Boshoff, A.; Blatch, G.L. Structure-function study of Plasmodium falciparum Hsp70 using three-dimensional modelling and in-vitro analyses. Protein Pept. Lett. 2008, 15, 1117-1125. [CrossRef] [PubMed] 
11. Zininga, T.; Achilonu, I.; Hoppe, H.; Prinsloo, E.; Dirr, H.W.; Shonhai, A. Overexpression, purification and characterization of the Plasmodium falciparum Hsp70-z (PfHsp70-z). PLoS ONE 2015, 10, e0129445. [CrossRef] [PubMed]

12. Zininga, T.; Makumire, S.; Gitau, G.W.; Njunge, J.M.; Pooe, O.J.; Klimek, H.; Scheurr, R.; Raifer, R.; Prinsloo, E.; Przyborski, J.M.; et al. Plasmodium falciparum Hop (PfHop) interacts with the Hsp70 chaperone in a nucleotide-dependent fashion and exhibits ligand selectivity. PLoS ONE 2015, 10, e0135326. [CrossRef] [PubMed]

13. Zininga, T.; Achilonu, I.; Hoppe, H.; Prinsloo, E.; Dirr, H.W.; Shonhai, A. Plasmodium falciparum Hsp70-z (Hsp110c) exhibits independent chaperone activity and interacts with Hsp70-1 in a nucleotide dependent fashion. Cell Stress Chaperones 2016, 21, 499-513. [CrossRef] [PubMed]

14. Zininga, T.; Pooe, O.J.; Makhado, P.B.; Ramatsui, L.; Achilinou, I.; Prinsloo, E.; Hoppe, H.; Dirr, H.; Shonhai, A. Polymyxin B inhibits the chaperone activity of Plasmodium falciparum Hsp70. Cell Stress Chaperones 2017. [CrossRef] [PubMed]

15. Singh, G.P.; Chandra, B.R.; Bhattacharya, A.; Akhouri, R.R.; Singh, S.K.; Sharma, A. Hyper-expansion of asparagines correlates with an abundance of proteins with prion-like domains in Plasmodium falciparum. Mol. Biochem. Parasitol. 2004, 137, 307-319. [CrossRef] [PubMed]

16. Pallavi, R.; Archarya, P.; Chandran, S.; Daily, J.P.; Tatu, U. Chaperone expression profiles correlate with distinct physiological states of Plasmodium falciparum in malaria patients. Malar. J. 2010, 9, 236. [CrossRef] [PubMed]

17. Shonhai, A.; Maier, A.G.; Przyborski, J.M.; Blatch, G.L. Intracellular protozoan parasites of humans: The role of molecular chaperones in development and pathogenesis. Protein Pept. Lett. 2011, 18, 143-157. [CrossRef] [PubMed]

18. Samie, A.; Housein, A.; Lall, N.; Meyer, J.J.M. Crude extracts of, and purified compounds from Pterocarpus angolensis, and the essential oil of Lippia javanica: Their in vitro cytotoxicities and activities against selected bacteria and Entamoeba histolytica. Ann. Trop. Med. Parasitol. 2009, 103, 427-439. [CrossRef] [PubMed]

19. Luseba, D.; Elgorashi, E.; Ntloedibe, D.; van Staden, J. Antibacterial, anti-inflammatory and mutagenic effects of some medicinal plants used in South Africa for the treatment of wounds and retained placenta in livestock. S. Afr. J. Bot. 2007, 73, 378-383. [CrossRef]

20. Mulaudzi, R.B.; Ndhlala, A.R.; Kulkarni, M.G.; Finnie, J.F.; Van Staden, J. Antimicrobial properties and phenolic contents of medicinal plants used by the Venda people for conditions related to venereal disease. J. Ethnopharmacol. 2011, 135, 330-337. [CrossRef] [PubMed]

21. Sigidi, M.; Anokwuru, C.; Zininga, T.; Tshisikhawe, M.P.; Shonhai, A.; Ramaite, I.; van der Venter, M.; Traoré, A.N.; Potgieter, N. Comparative in vitro cytotoxic, anti-inflammatory and anti-microbiological activities of two indigenous Venda medicinal plants. Trans. Med. Commun. 2016, 1, 9. [CrossRef]

22. Red List of South African Plants: Ziziphus Mucronata. Available online: http://redlist.sanbi.org/species. php?species=4058-4 (accessed on 2 June 2017).

23. Venter, F.; Venter, J.A. Making the Most of Indigenous Trees; Briza publications: Pretoria, South Africa, 1996; p. 300 .

24. Hutchings, A.; Haxton-Scott, A.; Lewis, G; Cunningham, A. Zulu Medicinal Plants-An Inventory Pietermaritizburg; University of Natal Press: Pietermaritzburg, South Africa, 1996.

25. Luthuli, S.D.; Chili, M.M.; Revaprasadu, N.; Shonhai, A. Cysteine-capped gold nanoparticles suppress aggregation of proteins exposed to heat stress. IUBMB Life 2013, 65, 454-461. [CrossRef] [PubMed]

26. Makler, M.T.; Hinrichs, D.J. Measurement of the lactate dehydrogenase activity of Plasmodium falciparum as an assessment of parasitemia. Am. J. Trop. Med. Hyg. 1993, 48, 205-210. [CrossRef] [PubMed]

27. Komlaga, G.; Cojean, S.; Dickson, R.A.; Beniddir, M.A.; Suyyagh-Albouz, S.; Mensah, M.L.K.; Loiseau, P.M. Antiplasmodial activity of selected medicinal plants used to treat malaria in Ghana. J. Parasitol. Res. 2016, 115, 3185-3195. [CrossRef] [PubMed]

28. Peatey, C.L.; Leroy, D.; Gardiner, D.L.; Trenholme, K.R. Anti-malarial drugs: How effective are they against Plasmodium falciparum gametocytes? Malar. J. 2012, 11, 34. [CrossRef] [PubMed]

29. Rahardja, F.; Tjokropranoto, R.; Widowati, W.; Lusiana, S.; Suhendra, A.; Tjahjani, S.; Budiman, I.; Maesaroh, M.; Fauziah, N. Phytochemical assay, potential of antimalarial and antioxidant activities of green tea extract and its fraction. J. Biomed. Eng. 2015, 1, 1-6. 
30. Lim, S.S.; Kim, H.-S.; Lee, D.-U. In vitro antimalarial activity of flavonoids and chalcones. Bull. Korean Chem. Soc. 2007, 28, 2495-2497.

31. Bankole, A.E.; Adekunle, A.A.; Sowemimo, A.A.; Umebese, C.E.; Abiodun, O.; Gbotosho, G.O. Phytochemical screening and in vivo antimalarial activity of extracts from three medicinal plants used in malaria treatment in Nigeria. Parasitol. Res. 2016, 115, 299-305. [CrossRef] [PubMed]

32. Cowan, M.M. Plant products as antimicrobial agents. Clin. Microbiol. Rev. 1999, 12, 546-582, PMCID:PMC88925. [PubMed]

33. Howarth, J.; Lloyd, D.G. Redox systems as conduits for antimalarial compounds. J. Antimicrob. Chemoth. 2001, 47, 122-124. [CrossRef]

34. Keller, C.C.; Kremsner, P.G.; Hittner, J.B.; Misukonis, M.A.; Weinberg, J.B.; Perkins, D.J. Elevated nitric oxide production in children with malarial anemia: Hemozoin-induced nitricoxide synthase type 2 transcripts and nitric oxide in blood mononuclear cells. Infect. Immun. 2004, 72, 4868-4873. [CrossRef] [PubMed]

35. Anokwuru, C.P.; Sigidi, M.T.; Zininga, T.; Tshisikhawe, M.P.; Shonhai, A.; Ramaite, I.D.I.; Traoré, A.N.; Potgieter, N. Phenolic contents, antioxidant activity and spectroscopic characteristics of Pterocarpus angolensis DC stem bark fractions. Indian J. Tradit. Know. 2017, 16, 400-406.

36. ICH Q2(R1) Validation of Analytical Procedures: Text and Methodology; ICH Harmonised Tripartite Guideline; ICH Secretariat: Switzerland, Geneva, 2005; Available online: http://www.gmp-compliance.org/ guidelines/gmp-guideline/ich-q2r1-validation-of-analytical-procedures-text-and-methodology (accessed on 28 June 2017).

37. Miller, J.N.; Miller, J.C. Statistics and Chemometrics for Analytical Chemistry, 6th ed.; Pearson Educational Limited: Edinburgh Gate, UK; pp. 110-151.

38. Trager, W.; Jensen, J.B. Human malaria parasites in continuous culture. Science 1997, 193, 673-675. [CrossRef]

Sample Availability: Samples of the compounds are available from the authors.

(C) 2017 by the authors. Licensee MDPI, Basel, Switzerland. This article is an open access article distributed under the terms and conditions of the Creative Commons Attribution (CC BY) license (http:/ / creativecommons.org/licenses/by/4.0/). 